\title{
HOMOTOPY METHOD FOR MINIMUM CONSUMPTION ORBIT TRANSFER PROBLEM*
}

\author{
Joseph Gergaud ${ }^{1}$ And Thomas HaBerkorn ${ }^{2}$
}

\begin{abstract}
The numerical resolution of the low thrust orbital transfer problem around the Earth with the maximization of the final mass or minimization of the consumption is investigated. This problem is difficult to solve by shooting method because the optimal control is discontinuous and a homotopic method is proposed to deal with these difficulties for which convergence properties are established. For a thrust of 0.1 Newton and a final time $50 \%$ greater than the minimum one, we obtain 1786 switching times.
\end{abstract}

Mathematics Subject Classification. 49M05, 65H20, 70Q05.

Received June 4, 2004. Revised February 14, 2005.

\section{INTRODUCTION}

The minimum time orbit transfer of a satellite around the Earth with low thrust has previously been investigated and solved by Caillau and Noailles [4]. Here, we are interested in the same problem with the final time fixed in three dimensions but with the maximization of the final mass, or the minimization of the consumption. To solve such a problem by shooting methods it is assumed, because the optimal control is discontinuous, that the structure of the optimal control is known [10]. The aim of this study is to construct a method to obtain the result without any a priori information on the optimal control.

The basic idea of the method we propose is to define a set of optimal control problems which depend on a parameter $\lambda \in[0,1]$ which connect the regular optimal control problem with minimization of the energy (square of $L^{2}$ norm of the control) for $\lambda=0$ to our problem with minimization of the consumption ( $L^{1}$ norm of the control) for $\lambda=1$. The Pontryagin Maximum Principle then provides a family of Boundary Value Problems and the shooting function associated to this family of $(B V P)_{\lambda}$ gives us a homotopy $S(z, \lambda)$. Following the path of the homotopy zeros will generate a solution of the problem. In particular, the number of switching times and their localization are found.

This paper is organized as follows: the optimal control formulation of the orbital transfer problem is described in Section 1. Sections 2 and 3 are devoted to the necessary condition and to the existence of solution. In Section 4 , the differentiability properties of the shooting function are studied and the numerical difficulties for

\footnotetext{
Keywords and phrases. Orbital transfer, optimal control problem, shooting method, differential homotopy, predictor-corrector method.

* Supported in part by the French Space Agency (CNES-ENSEEIHT contract 02/CNES/0257/00 - DPI 500.

1 ENSEEIHT-IRIT, CNRS-UMR 5505, 2 rue Camichel, BP 7122, 31071 Toulouse Cedex 7, France; gergaud@enseeiht.fr

2 Mathematics Department, 2565 Mc Carthy Mall, Honolulu HI, 96822, USA; haberkor@math.hawaii.edu
} 
solving this problem using shooting methods are analyzed. Section 5 explains the homotopy method and studies the convergence theorem. In Section 6 the Predictor-Corrector algorithm is presented. Finally, the numerical results are given in Section 7.

\section{Problem statement}

The satellite is considered as a material point and Cartesian coordinates are used. If $r(t) \in \mathbf{R}^{3}$ denotes the position, $v(t) \in \mathbf{R}^{3}$ the velocity, $m(t)$ the mass of the satellite and $T(t) \in \mathbf{R}^{3}$ the thrust of the engine, then the state equation is

$$
\begin{aligned}
\dot{r}(t) & =v(t) \\
\dot{v}(t) & =-\frac{\mu r(t)}{|r(t)|^{3}}+\frac{T(t)}{m(t)} \\
\dot{m}(t) & =-\beta|T(t)|,
\end{aligned}
$$

where $\mu$ is the gravitation constant of the Earth, $\beta$ is a positive constant and $|$.$| is the euclidian norm. There$ are also boundary constraints: the state is known at the initial time $(r(0), v(0), m(0))=\left(r_{0}, v_{0}, m_{0}\right)$ and must arrive in position and speed at the fixed final time $t_{f}$ in $\left(r\left(t_{f}\right), v\left(t_{f}\right)\right)=\left(r_{f}, v_{f}\right)$. An other constraint is that the thrust of the engine is limited

$$
|T(t)| \leq T_{\max }
$$

The objective is to maximize the final mass or, because of the state equation in the mass, to minimize the consumption of the engine

$$
\operatorname{Max} m\left(t_{f}\right) \Longleftrightarrow \operatorname{Min} \int_{0}^{t_{f}}|T(t)| \mathrm{d} t .
$$

If the control is standardized, $u(t)=T(t) / T_{\max }$, the optimal control problem can be expressed as

$$
(P)\left\{\begin{array}{l}
\operatorname{Min} \quad J(u)=\int_{0}^{t_{f}}|u(t)| \mathrm{d} t \\
\dot{r}(t)=v(t) \quad \text { a.e. in } \quad\left[0, t_{f}\right] \quad t_{f} \quad \text { fixed } \\
\dot{v}(t)=\Gamma(r(t))+\frac{T_{\max }}{m(t)} u(t) \\
\dot{m}(t)=-\beta T_{\max }|u(t)| \\
(r(t), v(t), m(t)) \in A \\
|u(t)| \leq 1 \\
r(0), v(0), m(0) \quad \text { fixed } \\
r\left(t_{f}\right), v\left(t_{f}\right) \quad \text { fixed }
\end{array}\right.
$$

with $\Gamma(r(t))=-\mu r(t) /|r(t)|^{3}$.

The two next sections are devoted to the necessary condition and to the existence of solution. For this, three assumptions are useful. First the satellite does not come too close to the Earth and does not use all its fuel:

(H1) the state stays in a security zone $A \in \mathbf{R}^{7}$,

$$
A=\left\{x=(r, v, m) \in \mathbf{R}^{7} \mid r>\rho_{0}>0, m>\chi_{0}>0\right\},
$$

where $\chi_{0}$ is the mass of the satellite without fuel.

Second, the system is controllable:

(H2) the final time $t_{f}$ is strictly greater than the minimum transfer time[4].

Eventually, the initial and final orbits are different:

(H3) the optimal control is not the null control.

We begin with the study of the necessary condition which will be helpful in proving the existence of solution. 


\section{NeCESSARY CONDITION}

The Hamiltonian of the problem is

$$
H\left(r, v, m, u, p_{0}, p_{r}, p_{v}, p_{m}\right)=\left(p_{0}-\beta T_{\max } p_{m}\right)|u|+\frac{T_{\max }}{m}\left(u \mid p_{v}\right)+\left(v \mid p_{r}\right)+\left(\Gamma(r) \mid p_{v}\right)
$$

where $p_{0} \geq 0$, and $p_{r}, p_{v}$ and $p_{m}$ are adjoint states in relation with $r, v$ and $m$. Under assumption (H1) the Pontryagin Maximum Principle [5] implies that there exists $p_{0} \geq 0$ and absolutely continuous functions $\left(p_{r}(t), p_{v}(t), p_{m}(t)\right)$ not simultaneously 0 which verify

(1) the adjoint equation

$$
\begin{aligned}
\dot{p}_{r}(t) & =-{ }^{t} \Gamma^{\prime}(r(t)) p_{v}(t) \\
\dot{p}_{v}(t) & =-p_{r}(t) \\
\dot{p}_{m}(t) & =\frac{T_{\max }}{m^{2}(t)}\left(u(t) \mid p_{v}(t)\right),
\end{aligned}
$$

(2) the transversality condition

$$
p_{m}\left(t_{f}\right)=0
$$

(3) the minimization of the Hamiltonian

$$
H\left(r, v, m, u, p_{0}, p_{r}, p_{v}, p_{m}\right)=\operatorname{Min}_{|\mathbf{w}| \leq 1} H\left(r, v, m, \mathbf{w}, p_{0}, p_{r}, p_{v}, p_{m}\right) \quad \text { a.e. }
$$

As in the case of the minimization of the final time [4], we have the following result.

Proposition 2.1. Under (H1), (H2) and (H3), $p_{v}$ has finitely many zeros.

Proof. If not, there exists a sequence $\left(t_{k}\right)_{k}, t_{k} \in\left[0, t_{f}\right]$, all distinct, such that $p_{v}\left(t_{k}\right)=0$. But $t_{f}$ is fixed, so there exists a subsequence, always noted $\left(t_{k}\right)_{k}$, which converges to $\bar{t}$. As $p_{v}$ is continuous, $p_{v}(\bar{t})=0$. But the adjoint equation (3) shows that $p_{v}$ is continuously differentiable, hence

$$
\frac{p_{v}\left(t_{k}\right)-p_{v}(\bar{t})}{t_{k}-\bar{t}}=0 \longrightarrow \dot{p}_{v}(\bar{t})=-p_{r}(\bar{t}) .
$$

So $p_{v}(\bar{t})=p_{r}(\bar{t})=0$ and the solution of the linear differential system $(2)$ and $(3)$ is $p_{r}(t)=p_{v}(t)=0$ for all $t \in\left[0, t_{f}\right]$. Then $p_{m}(t)=0$ for all $t$, because $\dot{p}_{m}(t)=0$ and $p_{m}\left(t_{f}\right)=0$, and $p_{0}$ must be strictly positive. In this case the minimization of the Hamiltonian, which is $H(u)=p_{0}|u|$, gives $u(t)=0$ a.e. This is impossible with the assumption (H3).

We can now prove that $p_{0}$ is not zero, and normalize it $\left(p_{0}=1\right)$.

Proposition 2.2. Under (H1), (H2) and (H3) $p_{0} \neq 0$.

Proof. If $p_{0}=0$ the Hamiltonian is

$$
H\left(r, v, m, u, p_{0}, p_{r}, p_{v}, p_{m}\right)=-\beta T_{\max } p_{m}|u|+\frac{T_{\max }}{m}\left(u \mid p_{v}\right)+\left(v \mid p_{r}\right)+\left(\Gamma(r) \mid p_{v}\right)
$$

The Proposition 2.1 implies that $p_{v}(t) \neq 0$ a.e. So, using the Cauchy-Schwarz inequality, it can be stated that

$$
H\left(r, v, u, p_{0}, p_{r}, p_{v}, p_{m}\right) \geq\left(-\frac{T_{\max }}{m(t)}\left|p_{v}\right|-\beta T_{\max } p_{m}\right)|u|+\text { terms without } u
$$


with an equality in the case $u(t)=-\alpha p_{v}(t), \alpha \geq 0$, so that the solution of the minimization of the Hamiltonian can be written $u(t)=-\alpha(y(t)) p_{v}(t) /\left|p_{v}(t)\right|$ a.e. with

$$
y(t)=\left(r(t), v(t), m(t), p_{r}(t), p_{v}(t), p_{m}(t)\right) \quad \text { and } \quad \alpha(y(t)) \in[0,1] .
$$

This implies that $\dot{p}_{m}(t)=-\alpha(y(t)) T_{\max }\left|p_{v}(t)\right| / m(t)^{2} \leq 0$ and, because $p_{m}\left(t_{f}\right)=0, p_{m}(t) \geq 0$ for all $t$.

Thus, to minimize the second member of $(5)$, it is necessary to have $|u(t)|=1$ a.e., and in this case the criterion $J(u)=\int_{0}^{t_{f}}|u(t)| \mathrm{d} t$ is equal to $t_{f}$ which is then the minimum time, but this is in contradiction with the assumption (H2).

Noting $y=(x, p)=\left(r, v, m, p_{r}, p_{v}, p_{m}\right)$ and defining the switching function

$$
\psi(y)=\psi\left(r, v, m, p_{r}, p_{v}, p_{m}\right)=1-\beta T_{\max } p_{m}-\frac{T_{\max }}{m}\left|p_{v}\right|,
$$

the minimization of the Hamiltonian can be solved.

Proposition 2.3. Under (H1), (H2) and (H3) and if $\left|p_{v}\right| \neq 0$ then the solution of the minimization of the Hamiltonian is

$$
u(y)=\left\{\begin{array}{lllll}
-\frac{p_{v}}{\left|p_{v}\right|} & & & \text { if } & \psi(y)<0 \\
-\alpha \frac{p_{v}}{\left|p_{v}\right|} & \text { with } & \alpha \in[0,1] & \text { if } & \psi(y)=0 \\
0 & & & \text { if } & \psi(y)>0
\end{array}\right.
$$

Remark 2.4. It is possible to study the case $\left|p_{v}\right|=0$ in the proposition as in [4], but this case was never encountered in our numerical experimentations and was not investigated.

\section{ExistenCE OF SOLUTION}

Under the assumption (H2) the set of admissible control is not empty and under the assumption (H1)

$$
|\dot{v}(t)| \leq \frac{\mu}{\rho_{0}^{2}}+\frac{T_{\max }}{\chi_{0}},
$$

then the set of admissible states is in a fixed compact of $\mathbf{R}^{n}$. So the only assumption not verified to apply the Filippov existence theorem, see for example Theorem (9.3.i) of [5], is the convexity assumption of $f(t, x, U)$, because of the state equation in the mass $\dot{m}(t)=-\beta T_{\max }|u(t)|$. To prove the existence of a solution the control can be rewritten, as in [10], in spherical coordinates, but we prefer to keep the original formulation. For this, generalized solutions (Gamkrelidze, Young), as define in [5] (pp. 18-22), are considered

$$
(P)_{G}\left\{\begin{array}{l}
\operatorname{Min} \quad J_{G}\left(\nu_{1}, \ldots, \nu_{n+2}, u^{(1)}, \ldots, u^{(n+2)}\right)=\int_{0}^{t_{f}} \sum_{j=1}^{n+2} \nu_{j}(t)\left|u^{(j)}(t)\right| \mathrm{d} t \\
\dot{r}(t)=v(t) \\
\dot{v}(t)=\Gamma(r(t))+\frac{T_{\max }}{m(t)} \sum_{j=1}^{n+2} \nu_{j}(t) u^{(j)}(t) \\
\dot{m}(t)=-\beta T_{\max } \sum_{j=1}^{n+2} \nu_{j}(t)\left|u^{(j)}(t)\right| \\
(r(t), v(t), m(t)) \in A \\
\left|u^{(j)}(t)\right| \leq 1 \\
\nu_{j}(t) \geq 0 \text { and } \sum_{j=1}^{n+2} \nu_{j}(t)=1 \\
r(0), v(0), m(0) \text { fixed } \\
r\left(t_{f}\right), v\left(t_{f}\right) \quad \text { fixed. }
\end{array}\right.
$$

For this generalized problem and if (H1) and (H2) are true, the Filippov existence theorem can be applied. 
Lemma 3.1. Under (H1), (H2) and (H3)

(1) for the generalized problem $(P)_{G}, p_{v}$ has finitely many zeros.

(2) Moreover,

$$
u(t)=\sum_{j=1}^{n+2} \bar{\nu}_{j}(t) \bar{u}^{(j)}(t)
$$

is a solution of our problem $(P)$, where $\left(\overline{\nu_{j}}, \bar{u}^{(j)}\right)_{j}$ is a solution of the generalized problem $(P)_{G}$.

Proof.

(1) The proof is the same as in Proposition 2.1.

(2) The minimization of the Hamiltonian for the generalized problem $(P)_{G}$ implies that when $\left|p_{v}(t)\right| \neq 0$ and $\bar{\nu}_{j}(t) \neq 0$, we have $\bar{u}^{(j)}(t)=-\alpha_{j}(y(t)) p_{v}(t) /\left|p_{v}(t)\right|$. So, it can be shown that for $u(t)$ defined as in (8), we have $|u(t)|=\sum_{j=1}^{n+2} \bar{\nu}_{j}(t)\left|\bar{u}^{(j)}(t)\right|$. Hence $u(t)$ is an admissible control for the problem $(P)$ and $J(u)$ has the same value as $J_{G}\left(\bar{\nu}_{1}, \ldots, \bar{\nu}_{n+2}, \bar{u}^{(1)}, \ldots, \bar{u}^{(n+2)}\right)$.

The existence of solution of $(P)$ readily follows.

Proposition 3.2. If (H1) and (H2) are true, then our optimal control problem (P) have a solution.

Proof. If (H3) is false, then $u(t)=0$ is trivially a solution, and if (H3) is true, then Lemma 3.1 gives the result.

\section{Shooting Method}

According to the Pontryagin Maximum Principle, extremals are solutions of the Boundary Value Problem

$$
(B V P)\left\{\begin{array}{l}
\dot{y}(t)=\varphi(y(t)) \quad t \in\left[0, t_{f}\right] \quad t_{f} \text { fixed } \\
r(0)=r_{0}, v(0)=v_{0}, m(0)=m_{0} \\
r\left(t_{f}\right)=r_{f}, v\left(t_{f}\right)=v_{f}, p_{m}\left(t_{f}\right)=0
\end{array}\right.
$$

with

$$
\varphi(y)=\left(v, \Gamma(r)+\frac{T_{\max }}{m} u(y),-\beta T_{\max }|u(y)|,-{ }^{t} \Gamma^{\prime}(r) p_{v},-p_{r}, \frac{T_{\max }}{m^{2}}\left(u(y) \mid p_{v}\right)\right) .
$$

The shooting function is in this case defined as follows:

$$
\begin{aligned}
S: \mathbf{R}^{n} & \longrightarrow \mathbf{R}^{n} \\
z & \longmapsto S(z)=\left(\left(y\left(t_{f}, z\right)\right)_{I_{f}}-y_{f}\right),
\end{aligned}
$$

with $y(., z)$ the solution of the Cauchy problem

$$
(I V P)_{z}\left\{\begin{array}{l}
\dot{y}(t)=\varphi(y(t)) \quad t \in\left[0, t_{f}\right] \quad t \text { fixed } \\
r(0)=r_{0}, v(0)=v_{0}, m(0)=m_{0} \\
p(0)=z
\end{array}\right.
$$

and

$$
\begin{aligned}
\left(y\left(t_{f}, z\right)\right)_{I_{f}} & =\left(r\left(t_{f}\right), v\left(t_{f}\right), p_{m}\left(t_{f}\right)\right), \\
y_{f} & =\left(r_{f}, v_{f}, 0\right) .
\end{aligned}
$$


The Boundary Value Problem $(B V P)$ can be solved by computing a zero of the shooting equation $S(z)=0$ using a Newton algorithm. This requires the differentiability of the shooting function which is not obvious here because the second member in the Initial Value Problem $(I V P)_{z}$ is not continuous. But with the definitions of

$$
\begin{aligned}
\Omega=\left\{z \in \mathbf{R}^{n} \mid\right. & x(t, z) \in A \quad \forall t \\
& p_{v}(t, z) \neq 0 \quad \forall t, \\
& \psi^{2}(y(t, z))+\left(p_{r}(t, z) \mid p_{v}(t, z)\right)^{2} \neq 0 \quad \forall t, \\
& \left.\psi(y(0, z)) \neq 0 \quad \text { and } \quad \psi\left(y\left(t_{f}, z\right)\right) \neq 0\right\}
\end{aligned}
$$

and

$$
\psi_{z}(t)=\psi(y(t, z))
$$

the regularity of the shooting function can be proved.

\section{Proposition 4.1.}

(1) For $z \in \Omega, \psi_{z}(t)$ is continuously differentiable and finitely many switching times exist such that $\psi(y(t, z))=0$

(2) $\Omega$ is an open subset of $\mathbf{R}^{n}$ and $S$ is $\mathrm{C}^{\infty}$ in $\Omega$.

Proof.

(1) Let $z \in \Omega$, then $p_{v}(t, z) \neq 0$ for all $t$ and the discontinuities of the control arrive only if $\psi(y(t, z))=0$, where $y(t, z)$ is an absolutely continuous function solution of

$$
(I V P)_{z}\left\{\begin{array}{l}
\dot{y}(t)=\varphi_{1}(y(t)) \quad \text { if } \quad \psi(y(t))<0 \\
\dot{y}(t)=\varphi_{2}(y(t)) \quad \text { if } \quad \psi(y(t))>0 \\
r(0)=r_{0}, \quad v(0)=v_{0}, \quad m(0)=m_{0} \\
p(0)=z,
\end{array}\right.
$$

$\varphi_{1}(y(t))=\varphi(y(t))$ with $u(t)=-\frac{p_{v}(t)}{\left|p_{v}(t)\right|}$ and $\varphi_{2}(y(t))=\varphi(y(t))$ with $u(t)=0$. However, here

$$
\dot{\psi}_{z}(t)=-\frac{T_{\max }}{m(t, z)} \frac{\left(p_{v}(t, z) \mid p_{r}(t, z)\right)}{\left|p_{v}(t, z)\right|}
$$

and the right member of (9) is continuous, thus $\psi_{z}(t)$ is absolutely continuous and $\psi_{z}(t)$ is $\mathrm{C}^{1}$. The definition of $\Omega$ implies that $\psi_{z}(t)=0$ and $\dot{\psi}_{z}(t)=0$ cannot be true simultaneously, so the times when $\psi_{z}(t)=0$ are isolated in the compact $\left[0, t_{f}\right]$ and $\psi_{z}(t)$ has a finite number of many zeros.

(2) Let $z \in \Omega$. When there is no time such that $\left|p_{v}(t, z)\right|=0$ and $\psi(y(t, z))=0$ the $(I V P)_{z}$ is a Cauchy problem with $\mathrm{C}^{\infty}$ second member and the result is obvious.

Suppose now there is only one switching time $\bar{t} \in] 0, t_{f}\left[\right.$ where $\psi_{z}(\bar{t})=0$. It can be assumed, without loss of generality that in $[0, \bar{t}[, y(t, z)$ is the solution of

$$
\left(I V P_{1}\right)_{z}\left\{\begin{array}{l}
\dot{y}(t)=\varphi_{1}(y(t)) \quad t \in[0, \bar{t}[ \\
r(0)=r_{0}, \quad v(0)=v_{0}, \quad m(0)=m_{0} \\
p(0)=z .
\end{array}\right.
$$

In this case one can prove that the function $z \mapsto \bar{t}(z)$ is well defined in a neighborhood of $z$ and is $\mathrm{C}^{\infty}$. For this the following function is considered:

$$
\begin{aligned}
F:[0, \bar{t}+\eta[\times B(z, \eta) & \longrightarrow \mathbf{R} \\
(t, w) & \longmapsto F(t, w)=\psi\left(y_{1}(t, w)\right) .
\end{aligned}
$$


This function is well defined for an $\eta>0$ and is $\mathrm{C}^{\infty}$ because $\varphi_{1}$ is $\mathrm{C}^{\infty}$ in $\left(I V P_{1}\right)_{z}$. However

$$
\frac{\partial F}{\partial t}(\bar{t}, z)=\dot{\psi}_{z}(t) \neq 0 \quad \text { in } \quad \Omega
$$

so the implicit function theorem can be applied and the function $\bar{t}(z)$ is $\mathrm{C}^{\infty}$ in a neighborhood of $z$.

We see now that the curve solution $y(t, z)$ is $y_{1}(t, z)$ in $[0, \bar{t}]$ and $y_{2}(t, z)$ in $\left[\bar{t}, t_{f}\right]$, where $y_{2}(t, z)$ is the solution of the initial value problem

$$
\left(I V P_{2}\right)_{z}\left\{\begin{array}{l}
\dot{y}(t)=\varphi_{2}(y(t)) \\
y(\bar{t})=y_{1}(\bar{t}, z)
\end{array}\right.
$$

This solution is transverse to the commutation surface $\psi(y)=0$ at $y(\bar{t}, z)$, i.e. that $\dot{y}_{1}(\bar{t}, z)$ and $\dot{y}_{2}(\bar{t}, z)$ are in the same side from the tangent plan of the commutation surface at $y_{1}(\bar{t}, z)$, because

$$
\dot{\psi}_{z}(t)=\frac{\partial \psi}{\partial y} \cdot \dot{y}_{1}(\bar{t}, z)=\frac{\partial \psi}{\partial y} \cdot \dot{y}_{2}(\bar{t}, z)=-\frac{T_{\max }}{m(\bar{t}, z)} \frac{\left(p_{v}(\bar{t}, z) \mid p_{r}(\bar{t}, z)\right)}{\left|p_{v}(\bar{t}, z)\right|}
$$

is different from 0 for $z \in \Omega$. Therefore the solution will be well defined in a neighborhood of $z$, and by composition the shooting function will also be $\mathrm{C}^{\infty}$. For the general case where there is a finite number of switching times, the argument is repeated.

Remark 4.2. To say that $z \in \Omega$ implies that there is in $\left[0, t_{f}\right]$ no time where a thrust arc appears or disappears when $z$ changes locally and that there is no singular arc.

To illustrate this result the simple problem $(Q)$ is considered where the acceleration of a material point $\ddot{x}(t)=u(t)$ is controlled, with the constraint on the control $|u(t)| \leq 1$, fixed final time, fixed initial and final states (in position and velocity) and a minimum cost $\int_{0}^{t_{f}}|u(t)| \mathrm{d} t$ is required. When applied to this problem, the Pontryagin Maximum Principle gives a Boundary Value Problem $(B V Q), \dot{x}_{1}(t)=x_{2}(t), \dot{x}_{2}(t)=u(t), \dot{p}_{1}(t)=$ $0, \dot{p}_{2}(t)=-p_{1}(t)$, the same two points conditions than the problem $(Q)$ and the relation between the control and the adjoint state

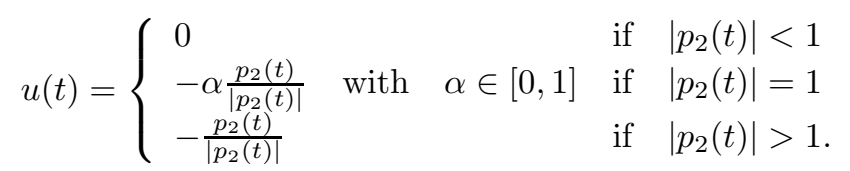

For this simple problem, it is easy to see that the set $\Omega$ where the shooting function is $\mathrm{C}^{\infty}$ is the union of 9 open sets of $\mathbf{R}^{2}$ defined by the structure of the control (see Fig. 1).

The numerical difficulties for solving the shooting equation $S(z)=0$ by a Newton algorithm are now analyzed. In fact, for our simple problem $(Q)$, it can be seen that:

(1) the shooting function is constant in $\mathcal{D}_{-1}, \mathcal{D}_{0}$ and $\mathcal{D}_{+1}$;

(2) the shooting function is not defined at $z=(0,-1)$ and $z=(0,1)$;

(3) the shooting function is not differentiable on the boundary of $\Omega$.

It is therefore evident for this simple example that if the initial point $z_{0}$ for solving the equation $S(z)=0$ is not in the correct domain, that is in the domain where the structure of the control is the same as the structure of the optimal control, then the Newton algorithm diverges. For the orbital transfer problem, if it is supposed that $p_{v}(t ; z) \neq 0$ everywhere, the situation is similar. In fact, $\psi(y(\bar{t}, z))=0$ and $\left(p_{r}(\bar{t}, z) \mid p_{v}(\bar{t}, z)\right)=0$ means that at $\bar{t}, y(t, z)$ is tangent to the commutation surface and thus that the structure of the control changes in $z$ or that we have a singular arc. However in practice the structure of the optimal control is not known, and the goal is to find the solution without this information. Another difficulty using Newton algorithm is to compute the Jacobian of the shooting function. In fact the second member of the Initial Value Problem (IVP) 


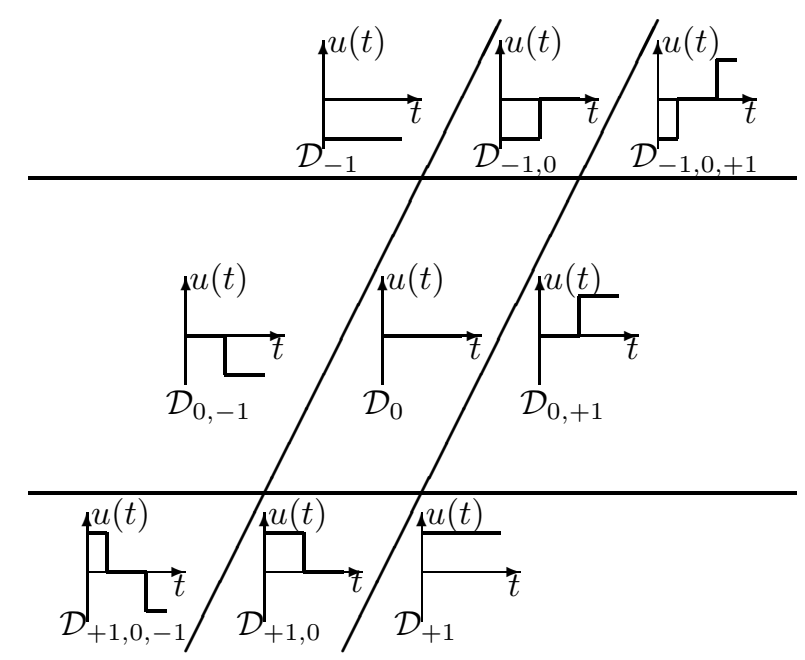

FIGURE 1. Stucture of the control with respect to $z$ for the problem $(Q)$; equations of lines are $z_{2}=-1, z_{2}=+1,-2 z_{1}+z_{2}=-1,-2 z_{1}+z_{2}=+1$.

which defines the shooting function is discontinuous. Therefore, it is necessary to compute the Jacobian by finite differences. For this, there must be an accurate adequation between the step used to compute the finite differences and the local error used for controlling the step in the integrator subroutine. If the step of finite differences is too small, then the Jacobian becomes numerically singular.

\section{Hомотору Method}

The principal idea of the method we propose is to define a family of problems which depend on a parameter $\lambda \in[0,1]$ and which connect the orbital transfer problem with the minimization of the energy for $\lambda=0$ to the problem with the minimization of the consumption for $\lambda=1[6]$. To this end, we only change the criterion to minimize

$$
(P)_{\lambda}: \operatorname{Min} \int_{0}^{t_{f}} \lambda|u(t)|+(1-\lambda)|u(t)|^{2} \mathrm{~d} t .
$$

Similar results, with same proofs, as those obtained in Sections 2 and 3 for the initial optimal control problem $(P)$ are proved for this family of problem.

Proposition 5.1. If (H1), (H2) and (H3) are true then

(1) $p_{\lambda v}$ has finitely many zero and $p_{0} \neq 0$;

(2) $(P)_{\lambda}$ has a solution for all $\lambda \in[0,1]$.

When the Pontryagin Maximum Principle is applied to this family of problems, a family of Boundary Value Problems $(B V P)_{\lambda}$ is obtained which is identical to $(B V P)$ except in the relation between the control and the adjoint state given by the minimization of the Hamiltonian.

Proposition 5.2. Under (H1), (H2) and (H3), the solution of the minimization of the Hamiltonian associated to the problem $(P)_{\lambda}$ in the case where $\left|p_{v}\right| \neq 0$ is for $\lambda \in[0,1[$

$$
u(y, \lambda)= \begin{cases}-\frac{p_{v}}{\left|p_{v}\right|} & \text { if } \psi(y)<-(1-\lambda) \\ \left(\frac{\psi}{2(1-\lambda)}-\frac{1}{2}\right) \frac{p_{v}}{\left|p_{v}\right|} & \text { if }|\psi(y)| \leq(1-\lambda) \\ 0 & \text { if } \psi(y)>(1-\lambda) .\end{cases}
$$

For $\lambda=1$ the relation is given by (7). 
Let us now define $y(t, z, \lambda)$ the solution of the initial value problem $(I V P)_{(z, \lambda)}$ associated to the boundary value problem $(B V P)_{\lambda}$

where

$$
(I V P)_{(z, \lambda)}\left\{\begin{array}{l}
\dot{y}(t)=\varphi(y(t), \lambda) \\
r(0)=r_{0}, v(0)=v_{0}, m(0)=m_{0} \\
p(0)=z
\end{array}\right.
$$

$$
\varphi(y, \lambda)=\left(v, \quad \Gamma(r)+\frac{T_{\max }}{m} u(y, \lambda), \quad-\beta T_{\max }|u(y, \lambda)|, \quad-{ }^{t} \Gamma^{\prime}(r) p_{v}, \quad-p_{r}, \quad \frac{T_{\max }}{m^{2}}\left(u(y, \lambda) \mid p_{v}\right)\right),
$$

the shooting homotopy $S(z, \lambda)=\left(y(t, z, \lambda)_{I_{f}}-y_{f}\right)$, that is the shooting function associated to the Boundary Value Problem $(B V P)_{\lambda}$, and the sets

$$
\begin{aligned}
& \Omega_{0}=\left\{(z, \lambda) \in \mathbf{R}^{n} \times\left[0,1\left[\mid x(t, z, \lambda) \in A \quad \forall t, \quad p_{v}(t, z, \lambda) \neq 0 \quad \forall t\right\},\right.\right. \\
& \Omega_{1}=\left\{(z, \lambda) \in \mathbf{R}^{n} \times[0,1] \mid x(t, z, \lambda) \in A \quad \forall t, \quad p_{v}(t, z, \lambda) \neq 0 \quad \forall t\right\},
\end{aligned}
$$

and

$$
\begin{gathered}
\Omega_{0}^{\prime}=\left\{z \in \Omega_{0} \mid(\psi(y(t, z, \lambda))-(1-\lambda))^{2}+\left(p_{r}(t, z, \lambda) \mid p_{v}(t, z, \lambda)\right)^{2} \neq 0 \quad \forall t\right. \\
(\psi(y(t, z, \lambda))+(1-\lambda))^{2}+\left(p_{r}(t, z, \lambda) \mid p_{v}(t, z, \lambda)\right)^{2} \neq 0 \quad \forall t, \\
\left.|\psi(y(0, z, \lambda))| \neq 1-\lambda \text { and }\left|\psi\left(y\left(t_{f}, z, \lambda\right)\right)\right| \neq 1-\lambda\right\} .
\end{gathered}
$$

It is now possible to prove regularity results of our shooting homotopy.

\section{Proposition 5.3.}

(1) $S(z, \lambda)$ is a continuous function in $\Omega_{0}$ and an upper semi-continuous set valued map in $\Omega_{1}$.

(2) The shooting homotopy $S(z, \lambda)$ is $\mathrm{C}^{\infty}$ in $\Omega_{0}^{\prime}$.

Proof.

(1) For $(z, \lambda) \in \Omega_{0}$, the function $\varphi(y, \lambda)$ is, by Proposition 5.2 , Lipschitz in $(y, \lambda)$. Thus $S(z, \lambda)$ is continuous in $\Omega_{0}$. For the case $(z, 1) \in \Omega_{1}$, the result is less obvious because, in this case the second member of the initial value problem $(I V P)_{(z, \lambda)}$ is in fact a differential inclusion. However, it can be seen that the second member of this differential inclusion has convex compact values and is upper semi-continuous. Then, known theorem of differential inclusion (see Th. 1 of Chap. 2 of [2]) gives the result.

(2) To prove that the shooting homotopy is $\mathrm{C}^{\infty}$ in $\Omega_{0}^{\prime}$ we build a new initial value problem, which can also be defined in the case $\lambda_{0}<0$ with the same relation (10) for the control without difficulties

$$
\left(I V P^{\prime}\right)_{(z, \lambda)}\left\{\begin{array}{l}
\dot{y}(t)=\varphi(y(t), \lambda) \\
\dot{\lambda}(t)=0 \\
r(0)=r_{0}, v(0)=v_{0}, m(0)=m_{0} \\
p(0)=z \\
\lambda(0)=\lambda_{0} .
\end{array}\right.
$$

Then, as in Proposition 4.1 we have to prove that the trajectory $y(t, z, \lambda)$ is transverse in the surfaces $\psi(y)=1-\lambda$ and $\psi(y)=-(1-\lambda)$. This comes immediately from the fact that

$$
\frac{\partial \psi}{\partial y}(y(t, z, \lambda)) \cdot \dot{y}(t, z, \lambda)=-\frac{1}{2(1-\lambda)} \frac{T_{\max }}{m(t, z, \lambda)} \frac{\left(p_{v}(t, z, \lambda) \mid p_{r}(t, z, \lambda)\right)}{\left|p_{v}(t, z, \lambda)\right|}
$$

is not zero when $|\psi(y(t, z, \lambda))|=1-\lambda$ and $(z, \lambda)$ is in $\Omega_{0}^{\prime}$. 
Remark 5.4. The smoothness of the shooting homotopy at $(z, 1)$ is a more delicate problem. In fact, it is also possible to define the problem $\left(I V P^{\prime}\right)_{(z, \lambda)}$ for $\lambda>1$, but the minimization of the Hamiltonian gives in this case, always for $\left|p_{v}\right| \neq 0$,

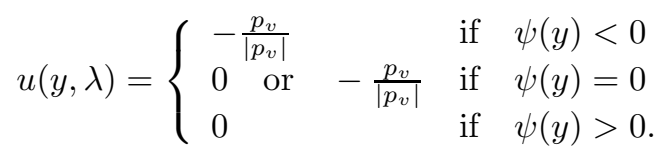

So, when $\lambda \geq 1$, the shooting homotopy is constant with respect to $\lambda$ for $z$ fixed in $\Omega$. It is then not obvious that the partial derivative with respect to $\lambda$ of the shooting homotopy at point $(z, 1)$ is zero. However, For a numerical point of view, this is not a problem because we will not have to compute partial derivatives with respect to $\lambda$ at such points $(z, 1)$.

Corollary 5.5. For $(z, \lambda) \in \Omega_{0}$, the control $u(t, z, \lambda)$ is piecewise $\mathrm{C}^{1}$ in $t$.

Moreover an interesting property can be indicated.

Proposition 5.6. Under assumptions (H1),(H2) and (H3) and if $\left(x_{\lambda}, u_{\lambda}\right)$ is a solution of $(P)_{\lambda}$, then for $0 \leq \lambda \leq \lambda^{\prime} \leq 1$ there are:

(1) $J_{\lambda}\left(u_{\lambda}\right) \leq J_{\lambda^{\prime}}\left(u_{\lambda^{\prime}}\right) \leq J_{1}\left(u_{1}\right) \leq J_{1}\left(u_{\lambda}\right)$;

(2) $\left|J_{1}\left(u_{\lambda}\right)-J_{\lambda}\left(u_{\lambda}\right)\right| \rightarrow 0$ when $\lambda \rightarrow 1$;

(3) $J_{\lambda}\left(u_{\lambda}\right) \rightarrow J_{1}\left(u_{1}\right)$ and $J_{1}\left(u_{\lambda}\right) \rightarrow J_{1}\left(u_{1}\right)$ when $\lambda \rightarrow 1$.

Proof.

(1) For all $u \in B(0,1)$ and $0 \leq \lambda \leq \lambda^{\prime} \leq 1$

$$
\begin{aligned}
\lambda^{\prime}|u|+\left(1-\lambda^{\prime}\right)|u|^{2} & =\lambda|u|+(1-\lambda)|u|^{2}+\left(\lambda^{\prime}-\lambda\right)\left(|u|-|u|^{2}\right) \\
& \geq \lambda|u|+(1-\lambda)|u|^{2} .
\end{aligned}
$$

This implies that $J_{\lambda}(u) \leq J_{\lambda^{\prime}}(u)$ for all admissible control. As the sets of admissible control are the same for all $\lambda \in[0,1]$ the following inequality is obtained

$$
J_{\lambda}\left(u_{\lambda}\right) \leq J_{\lambda}\left(u_{\lambda^{\prime}}\right) \leq J_{\lambda^{\prime}}\left(u_{\lambda^{\prime}}\right)
$$

(2) The function $l(u, \lambda)=\lambda|u|+(1-\lambda)|u|^{2}$ is continuous on the compact $B(0,1) \times[0,1]$. So it is uniformly continuous. Thus for all $\varepsilon>0$, there exists $\eta>0$, such that for all $\lambda, \quad|\lambda-1|<\eta$ and $u \in B(0,1)$

$$
|l(u, \lambda)-l(u, 1)|<\varepsilon .
$$

Hence

$$
\begin{aligned}
\left|J_{\lambda}\left(u_{\lambda}\right)-J_{1}\left(u_{\lambda}\right)\right| & \leq \int_{0}^{t_{f}}\left|l\left(u_{\lambda}(t), \lambda\right)-l\left(u_{\lambda}(t), 1\right)\right| \mathrm{d} t \\
& \leq \varepsilon t_{f} .
\end{aligned}
$$

(3) The result is obvious.

It is of course interesting to study the convergence of a sequence of solution of problems $(P)_{\lambda_{k}}$, noted $\left(x_{k}, u_{k}\right)_{k}$, when $\left(\lambda_{k}\right)_{k}$ converges to 1 . 
Proposition 5.7. If (H1) and (H2) are true, $\left(\lambda_{k}\right)_{k}$ is a sequence of [0,1] which converges to 1 when $k$ converges to $+\infty$ and $\left(x_{k}, u_{k}\right)_{k}$ is for all $k$ an optimal pair of $(P)_{\lambda_{k}}$, then there exists a subsequence, always noted $\left(x_{k}, u_{k}\right)_{k}$, which converges to a solution $(\bar{x}, \bar{u})$ of the problem $(P)$ in the following sense:

(1) $x_{k} \rightarrow \bar{x}$ uniformly on $\left[0, t_{f}\right]$;

(2) $u_{k} \rightarrow \bar{u}$ weakly-* in $L_{m}^{\infty}\left(\left[0, t_{f}\right]\right)$.

Proof.

(1) The proof follows the lines of the classical Filippov theorem. First, because of the proposition 5.6, $u_{k}$ is a minimized sequence of $(P)$. Secondly the assumptions ensure that $\left(x_{k}\right)_{k}$ is absolutely continuous and equibounded and that $\left\|\dot{x}_{k}\right\|_{\infty} \leq l \quad \forall k$. The Theorem 4 page 13 of [2] says that there exists a subsequence, always noted $\left(x_{k}\right)_{k}$, which converges uniformly to $\bar{x}$ absolutely continuous and $\left(\dot{x}_{k}\right)_{k}$ converges weakly-* to $\dot{\bar{x}}$ in $L_{n}^{\infty}\left(\left[0, t_{f}\right]\right)$. From the lower closure Theorem (8.8.i) of [5], $\dot{\bar{x}}(t)$ belongs to $\tilde{Q}_{G}(\bar{x}(t))$ almost everywhere. The set $\tilde{Q}_{G}(x(t))$ is here, as defined at page 313 of [5], the set associated to the generalized problem $(P)_{G}$ of all $\left(\tilde{x}_{0}, \tilde{x}\right) \in \mathbf{R}^{n+1}$ with $\tilde{x}_{0} \geq l_{0}\left(\nu_{1}, \ldots, \nu_{n+2}, u^{(1)}, \ldots, u^{(n+2)}\right)$ and $\tilde{x}=f_{G}\left(x, \nu_{1}, \ldots, \nu_{n+2}, u^{(1)}, \ldots, u^{(n+2)}\right)$ for some generalized control $\left(l_{G}\right.$ and $f_{G}$ are the functions which define respectively the cost and the state equation of the generalized problem). Finally, $\bar{x}$ is an optimal trajectory of the generalized problem $(P)_{G}$. By measurable selection, a generalized control $\left(\bar{\nu}_{1}, \ldots, \bar{\nu}_{n+2}, \bar{u}^{(1)}, \ldots, \bar{u}^{(n+2)}\right)$ associated to $\bar{x}$ can be chosen. Now, as in Section 3 , if $\bar{u}(t)=\sum_{j=1}^{n+2} \bar{\nu}_{j}(t) u^{(i)}(t)$, then $\bar{u}$ is an admissible control of the problem $(P)$ associated to $\bar{x}$.

(2) From the state equation it can be observed that

$$
u_{k}(t)=\frac{m_{k}(t)}{T_{\max }}\left(\dot{v}_{k}(t)-\Gamma\left(r_{k}(t)\right)\right)
$$

However $\left(v_{k}\right)_{k}$ and $\left(m_{k}\right)_{k}$ converge uniformly to $\bar{v}$ and $\bar{m}$ and $\left(\dot{v}_{k}\right)_{k}$ converges weakly-* to $\dot{\bar{v}}$, and the result is obtained.

Remark 5.8. The proposition is also true if the optimal solution of the initial optimal control problem has a singular arc. The singular arc in our numerical experimentation was not encountered, however the existence of such arcs for particular initial and terminal orbits is an open question.

\section{Algorithm}

The computation of the zero path of our homotopy $S(z, \lambda)$ is now considered. The simplest way to follow this zero path is basically to try to solve a sequence of equations of the form $S(z, \lambda)=0$, with $\lambda$ growing from 0 to 1 , by taking the previously obtained solution as an initial guess for the next try. This algorithm converges easily for the simple problem $(Q)$, unfortunately an appropriate sequence of $\left(\lambda_{k}\right)_{k}$ for our optimal transfer problem could not be found. As values approaching $\lambda=0.8$ are used, the Newton algorithm diverges. So we use a Predictor-Corrector (PC) method or continuation method which dynamically computes the $\lambda$ values. In order to have a self-contained article and to insist on numerical implementation difficulties, the basic idea of this algorithm is recalled. For more detailed explanation of the PC method, see $[1,11,12]$.

Assume that the considered homotopy $S(z, \lambda)$ is regular enough $\left(\mathcal{C}^{2}\right)$, and that the zero path which comes from $\left(z_{0}, 0\right)$ is a differentiable curve $C$. In that case it is possible to parameterize this curve with respect to the arclength $s$. Now, if it is supposed that $S^{\prime}(z(s), \lambda(s))$ is of full rank on the curve $S(z(s), \lambda(s))=0$, then the two unit tangent vectors to $C$ at $s$ are the two elements of the kernel of $S^{\prime}(z(s), \lambda(s))$ of norm 1 . To determine the direction, the augmented Jacobian matrix is introduced

$$
A(s)=\left(\begin{array}{cc}
\frac{\partial z}{\partial s}(s) & \frac{\partial \lambda}{\partial s}(s) \\
\frac{\partial S}{\partial z}(z(s), \lambda(s)) & \frac{\partial S}{\partial \lambda}(z(s), \lambda(s))
\end{array}\right) .
$$


Because $S^{\prime}(z(s), \lambda(s))$ is supposed of full rank, this matrix is non singular and so the sign of its determinant is constant

$$
\operatorname{sgn}(\operatorname{det}(A(s)))=\operatorname{sgn}(\operatorname{det}(A(0))) .
$$

Hence, by setting the first direction of the tangent vector (we take $\partial \lambda / \partial s(0)>0$ ), the unique unit tangent vector to $C$ verifying (12) can be computed. We note $\phi\left(S^{\prime}(z, \lambda)\right)$ this tangent vector.

Now, following the zero path of $S$ is equivalent to the integration of the initial value problem $(I V P)$

$$
(I V P)\left\{\begin{array}{l}
(\dot{z}(s), \dot{\lambda}(s))=\phi\left(S^{\prime}(z(s), \lambda(s))\right) \\
(z(0), \lambda(0))=\left(z_{0}, 0\right)
\end{array}\right.
$$

The HOMPACK90 software [12] has been implemented to integrate the (IVP) problem. The algorithm developed in this software uses the fact that $S(z(s), \lambda(s))=0$. An integration step is decomposed into two main phases: a prediction and a correction. The prediction step consists of a simple scheme, for instance the Euler scheme:

$$
w^{n+1}=\left(z^{n}, \lambda^{n}\right)+h \phi\left(S^{\prime}\left(z^{n}, \lambda^{n}\right)\right)(h \text { is the steplength }) .
$$

The correction phase consists in getting back onto the zero path which should not be too far:

$$
\left(z^{n+1}, \lambda^{n+1}\right)=\underset{S(\omega)=0}{\operatorname{argmin}} \frac{1}{2}\left\|\omega-w^{n+1}\right\|^{2} .
$$

This correction is performed with Newton steps, which is supposed not be too expensive as the solution is near.

The main advantage of this method is that the steplength of the prediction can take into account the previous predictions so that if the zero path is regular, the software reaches $\lambda=1$ very rapidly. However there is a drawback as for each prediction and correction step, the Jacobian of the homotopy has to be evaluated. But, because for $\lambda=1$ the control is discontinuous, we can only approximate the Jacobian by finite differences (it is not possible to use here variational equation or automatic differentiation). This requires a good adequation between the integration step error for computing the shooting homotopy and the step of finite differences. This is a crucial point for the numerical convergence. Of course, the step of finite differences must not be too large. However it must not be also too small in comparison with the integration step error, otherwise we "differentiate" numerical errors.

\section{Numerical ReSults}

As in $[4,7,9]$, because of the large number of revolutions and strong oscillations in Cartesian coordinates, the Gauss coordinates system which describes the movement of the satellite in a more orbit-related point of view is preferred. There we use the first five components of the state vector to characterize the osculating orbit (the orbit the satellite would follow if no thrust was applied), while the sixth component indicates the current position of the satellite on this orbit. As the orbit deformation is quite smooth, especially for low thrust transfers, this guarantees a very good numerical stability for our state vector, which would not be the case with the Cartesian expression. This particular choice of coordinates is illustrated by Figure 2.

The state variables $(x, m)=\left(P, e_{x}, e_{y}, h_{x}, h_{y}, L, m\right)$ in $\mathbf{R}^{7}$ are now defined:

- semi-latus rectum $P$;

- eccentricity vector $\left(e_{x}, e_{y}\right)$, in the orbit plane, oriented towards perigee;

- rotation vector $\left(h_{x}, h_{y}\right)$, in the equatorial plane, collinear to the intersection of orbit and equatorial planes;

- cumulative longitude $L$;

- mass $m$; 


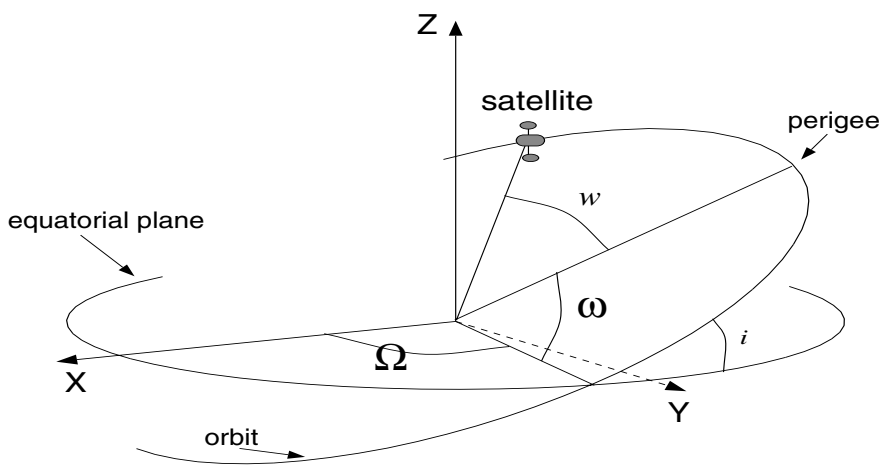

Figure 2. Orbital parameters: $P$ is the semi-latus rectum, $w$ is the true anomaly, $\Omega$ the ascending node longitude, $\omega$ the argument of perigee and $i$ the inclination with respect to equatorial plane.

with

$$
\begin{array}{ll}
e_{x}=e \cos (\Omega+\omega) \quad, \quad e_{y}=e \sin (\Omega+\omega), \\
h_{x}=\tan (i / 2) \cos \Omega \\
L=\Omega+\omega+\mathrm{w} .
\end{array}
$$

The tridimensional control is expressed in the mobile referential attached to the satellite $u=(q, s, w)$, where $q=r /|r|$ is the radial thrust, $w=q \times \dot{q} /|q \times \dot{q}|$ the normal thrust and $s=w \times q$ the ortho-radial thrust.

If we note

$$
f_{0}(x)=\sqrt{\frac{\mu}{P}}\left(\begin{array}{c}
0 \\
0 \\
0 \\
0 \\
0 \\
\frac{W^{2}}{P}
\end{array}\right) \text { and } B(x)=\sqrt{\frac{P}{\mu}}\left(\begin{array}{ccc}
0 & 2 P / W & 0 \\
\sin L & \cos L+\left(e_{x}+\cos L\right) / W & -Z e_{y} / W \\
-\cos L & \sin L+\left(e_{y}+\sin L\right) / W & Z e_{x} / W \\
0 & 0 & \frac{C}{2} \cos L / W \\
0 & 0 & \frac{C}{2} \sin L / W \\
0 & 0 & Z / W
\end{array}\right)
$$

with

$$
\begin{aligned}
W & =1+e_{x} \cos L+e_{y} \sin L \\
Z & =h_{x} \sin L-h_{y} \cos L \\
C & =1+h_{x}^{2}+h_{y}^{2},
\end{aligned}
$$

the maximal final mass (or minimum fuel consumption) orbital transfer problem can be written

$$
\left(P_{m_{f}}\right)\left\{\begin{array}{l}
\operatorname{Min} \int_{0}^{t_{f}}|u(t)| \mathrm{d} t \\
\dot{x}(t)=f_{0}(x(t))+\frac{T_{\max }}{m(t)} B(x(t)) u(t) \quad \text { a.e. in } \quad\left[0, t_{f}\right] \quad t_{f} \quad \text { fixed } \\
\dot{m}(t)=-\beta T_{\max }|u(t)| \\
|u(t)| \leq 1 \\
x(0), m(0) \quad \text { fixed } \\
x\left(t_{f}\right) \text { fixed } \\
t_{f}=t_{f_{\min }} \cdot c_{t_{f}} .
\end{array}\right.
$$

The physical constants $\mu$ and $\beta$ are respectively $398600.47 \mathrm{~km}^{3} \mathrm{~s}^{-2}$ and $0.05112 \mathrm{~km}^{-1} \mathrm{~s}$. The initial and final states are $x(0)=(11625,0.75,0,0.0612,0, \pi), m(0)=1500$ and $x\left(t_{f}\right)=\left(42165,0,0,0,0, L_{f}\right), L_{f}$ fixed. The minimum transfer time $t_{f_{\min }}$ is obtained via tfmin software [3], and $c_{t_{f}}$ is a constant greater than 1 . 


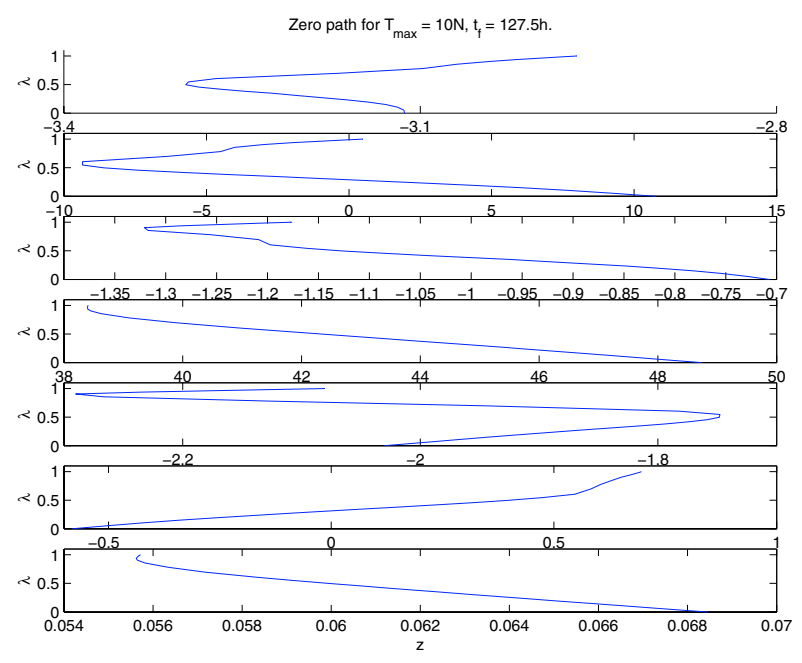

Figure 3. Path of zeros of $S(z, \lambda)$; from the top to the bottom $z_{1}=p_{P}(0), \ldots, z_{6}=p_{L}(0), z_{7}=$ $p_{m}(0)$ in abscissa.

Remark 7.1. This problem is slightly different from the problem $(P)$. In fact, $L_{f}$ fixed means that the position of the satellite on the final orbit and the number of revolutions are fixed. We can also easily see that:

(1) $p_{v}(t)=0 \Longleftrightarrow{ }^{t} B(x(t)) p(t)=0$, with $p(t)$ the adjoint state associated to $x(t)$;

(2) the expression of the switching function is here $\psi(y)=1-\beta T_{\max } p_{m}-\left.\left(T_{\max } / m\right)\right|^{t} B(x) p \mid$.

To apply our method, a solution of the problem with minimization of the energy must be found. In order to solve this initialization problem, another homotopy is introduced where the homotopic parameter $\lambda_{I C}$ is added in the initial conditions:

$$
(P)_{\lambda_{I C}}\left\{\begin{array}{l}
\min \int_{0}^{t_{f}}|u(t)|^{2} \mathrm{~d} t \\
\dot{x}(t)=f_{0}(x(t))+\frac{T_{\max }}{m(t)} B(x(t)) u(t) \quad \text { a.e. } \quad \text { in } \quad\left[0, t_{f}\right] \quad t_{f} \quad \text { fixed } \\
\dot{m}(t)=-\beta T_{\max }|u(t)| \\
|u(t)| \leq 1 \\
x_{0}, x_{f} \text { fixed } \\
t_{f}=t_{f_{\min }} \cdot c_{t_{f}} \\
x(0)=\left(1-\lambda_{I C}\right) x_{f}+\lambda_{I C} x_{0} \\
m(0) \text { fixed }
\end{array}\right.
$$

with $x_{0}=(11625,0.75,0,0.0612,0, \pi), \quad m(0)=1500$ and $x_{f}=\left(42165,0,0,0,0, L_{f}\right), \quad L_{f}$ fixed. It is easy to see that for $\lambda_{I C}=0$ the null control is a trivial and unique solution for the problem without rendez-vous $(i . e$. with the final cumulative longitude $L_{f}$ free). So the shooting function $S_{I C}(z, 0)$ associated with problem $(P)_{\lambda_{I C}}$ for $\lambda_{I C}=0$ can be numerically solved easily by Newton method with the initial point $z_{0}=(0,0,0,0,0,0,0)$. Then with a discrete continuation it is possible to find a zero of $S_{I C}(z, 1)$. Using this method, an initialization for a thrust of 0.1 Newton can be found. This method requires no preliminary knowledge of the solution (such as a solution for a greater thrust in the minimum time case).

The numerical results presented here have been obtained with the software $M f \max [8]$, based on the software HOMPACK $90[11,12]$ for computing the zero path of the homotopy $S(z, \lambda)$. We first consider the case of a thrust of $10 \mathrm{~N}$. Figure 3 plots the path of zeros of the homotopy $S(z, \lambda)$. We can see the necessity of using the PC method: the algorithm automatically controls the progress in the curvilinear abscissa and thus in the homotopic parameter, and the predictor step gives an accurate initial estimate for the corrector step. 

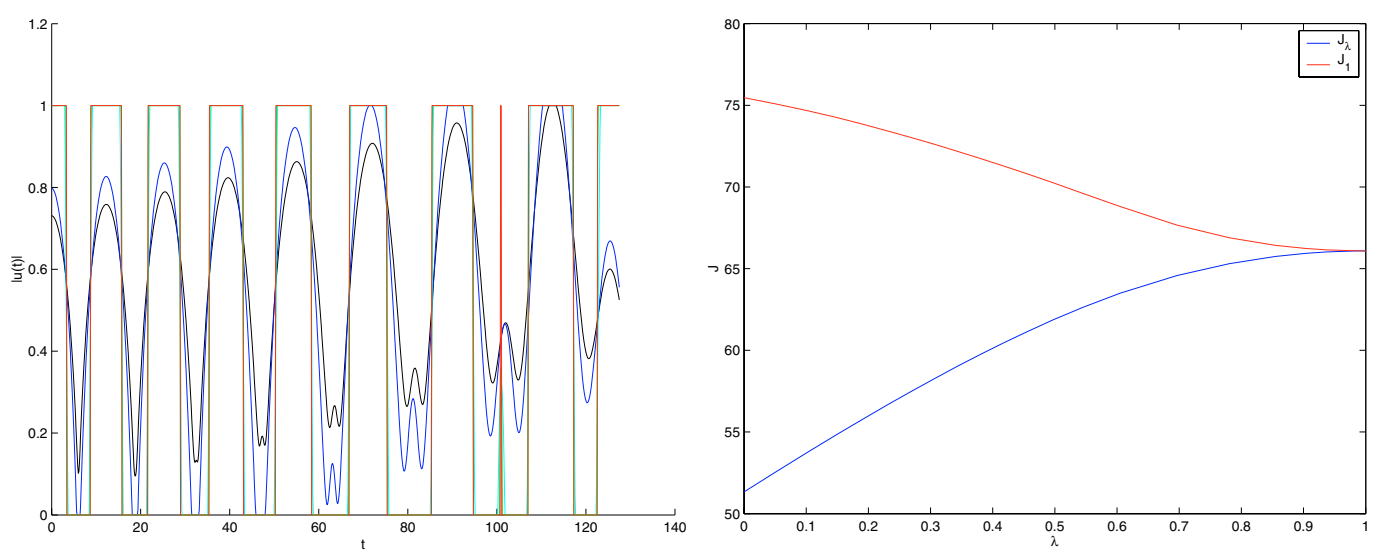

FiguRE 4 . The left hand figure shows the evolution of the norm of the control for $\lambda=0,0.38$ and 1 , and the right hand figure the costs $J_{\lambda}\left(u_{\lambda}\right)$ and $J_{1}\left(u_{\lambda}\right)$ with respect to $\lambda$.
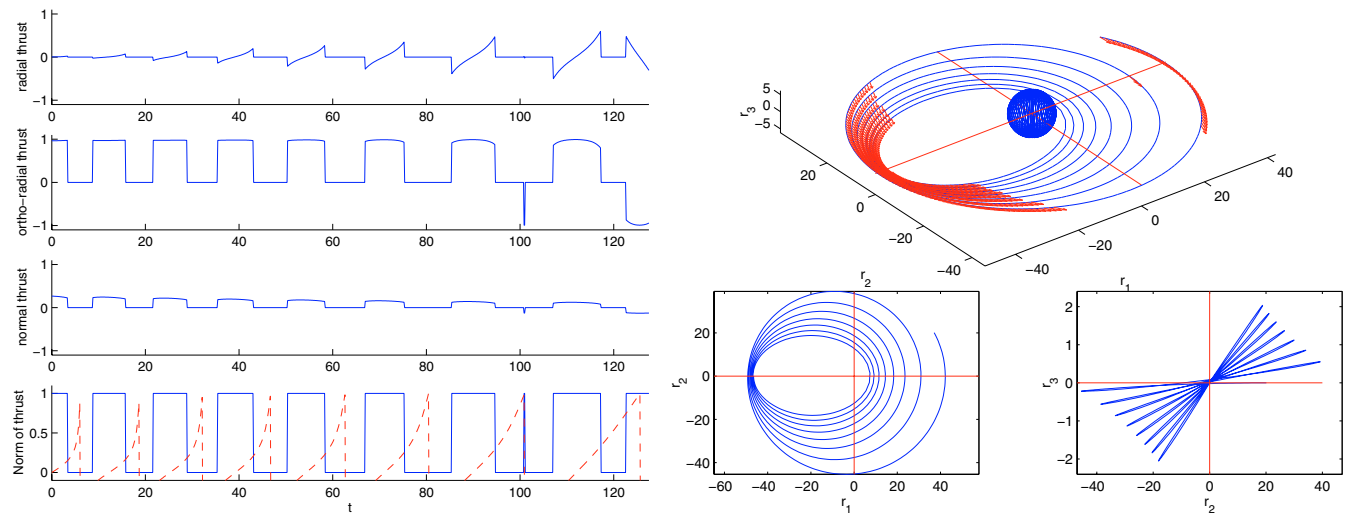

Figure 5. The left hand figure represents from the top to the bottom the three components $(q, s, w)$ and the norm of the optimal control, and the right hand figure represents the trajectory and thrust at solution.

The left part of the Figure 4 shows norms of three optimal control of $(P)_{\lambda}$ for $\lambda=0,0.38$ and 1 . This illustrates the convergence of the control sequence to the "bang-bang" optimal control when $\lambda$ converges to 1 . The right part of this figure is in relation with the properties in $J_{\lambda}$ of Proposition 5.6.

We of course verify numerically that there is no time where ${ }^{t} B(x(t)) p(t)=0$ and that the trajectory $y(t)$ is always transverses to the surface of commutation at switching times. For example the minimum value of the function $\sqrt{\psi_{z}(t)^{2}+\dot{\psi}_{z}(t)^{2}}$ on $\left[0, t_{f}\right]$ is 0.0086 . So we can now analyze ( $c f$. Fig. 5 ) the structure of the optimal control we obtained. We can observe that there is thrust arcs at all the apogees and at the 2 last perigees. The main thrust is ortho-radial and we have an inversion of the thrust between apogee and perigee ones. In all our numerical experimentations we have observed that thrust arcs are present at all apogees, at the last perigees (see for example the Figure 6 which represents the 3D trajectory for a thrust of $0.1 \mathrm{~N}$ and a final time $\left.t_{f}=1.5 \times t_{\min }\right)$ and sometimes at the first perigees.

We finish by giving in Table 1 the final mass and the number of switching times for thrusts from $10 \mathrm{~N}$ to $0.1 \mathrm{~N}$, final time $t_{f} 50 \%$ greater than the minimum time (which depends on the maximal thrust $T_{\max }$ ) and $L_{f}$ fixed. We can observe in this table that the final mass seems constant. 


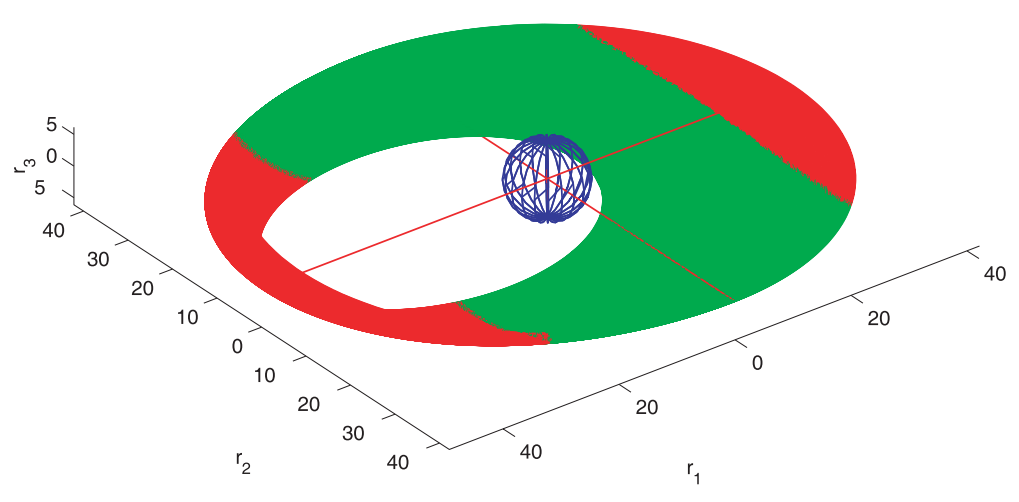

FiguRE 6. 3D-trajectory and thrust arcs for $0.1 \mathrm{~N}$.

TABLE 1. Optimal mass, number of revolutions $\left(L_{f}-L_{0}\right) / 2 \pi$ and number of switching times.

\begin{tabular}{|c|c|c|c|}
\hline$T_{\max }$ (Newton) & $\begin{array}{c}\text { Nb. of } \\
\text { revolutions }\end{array}$ & Mass & $\begin{array}{c}\text { Nb. of } \\
\text { switching times }\end{array}$ \\
\hline 10 & 7.3 & 1378.37 & 18 \\
5 & 15.1 & 1378.29 & 36 \\
2.5 & 30.1 & 1378.15 & 73 \\
1 & 75.4 & 1377.94 & 179 \\
0.5 & 150.7 & 1377.99 & 360 \\
0.2 & 376.8 & 1377.97 & 915 \\
0.1 & 753.7 & 1377.99 & 1786 \\
\hline
\end{tabular}

\section{Conclusion}

In conclusion, It has been shown that the solution of the orbital transfer problem with the maximization of the final mass can be computed without any information on that solution. In addition the numerical results were more accurate than expected (thanks to HOMPACK90 and to RKF45). This method has also been applied successfully to other initial states, with the final cumulative longitude free, and with the final time free and the final cumulative longitude fixed $[8,9]$. Although, even in all the numerical tests we considered, we did not find any time for which ${ }^{t} B(x(t)) p(t)=0$ or any singular arcs, we do not know if it can happened in some cases. Finally, it is clear that for free cumulative longitude and fixed final time the optimal mass $m\left(t_{f}\right)$ is increasing with respect to the final time, however we do not know if there exists a solution for free final cumulative longitude and free final time.

Acknowledgements. We thank the reviewers very much for their work and useful comments on this article, especially the enlightening remarks which have improved the form.

\section{REFERENCES}

[1] E.L. Allgower and K. Georg, Numerical Continuation Method. An Introduction. Springer-Verlag, Berlin (1990).

[2] J.-P. Aubin and A. Cellina, Differential Inclusion. Springer-Verlag (1984).

[3] J.-B. Caillau, J. Gergaud and J. Noailles, TfMin Short reference manual. Rapport de recherche RT/APO/01/3, ENSEEIHT-IRIT, UMR CNRS 5505, 2 rue Camichel, 31071 Toulouse, France, juillet 2001. http://www.enseeiht.fr/ caillau/papers/rt-01-3.html

[4] J.-B. Caillau and J. Noailles, Coplanar control of a satellite around the Earth. ESAIM: COCV 6 (2001) 239-258.

[5] L. Cesari, Optimization - Theory and Applications. Springer-Verlag (1983). 
[6] J. Gergaud, Résolution numérique de problèmes de commande optimale à solution Bang-Bang par des méthodes homotopiques simpliciales. Ph.D. Thesis, ENSEEIHT, Institut National Polytechnique de Toulouse, France (janvier 1989).

[7] J. Gergaud, T. Haberkorn and P. Martinon, Low thrust minimum-fuel orbital transfer: a homotopic approach. J. Guidance, Control, and Dynamics 27 (2004) 1046-1060.

[8] J. Gergaud, T. Haberkorn and J. Noailles, Mfmax(v0 and v1): Method explanation manual. Rapport de recherche RT/APO/04/1, ENSEEIHT-IRIT, UMR CNRS 5505, 2 rue Camichel, 31071 Toulouse, France, january 2004. http://www.enseeiht.fr/apo/mfmax/

[9] T. Haberkorn, Transfert orbital à poussée faible avec minimisation de la consommation: résolution par homotopie différentielle. Ph.D. Thesis, ENSEEIHT, Institut National Polytechnique de Toulouse, France (octobre 2004).

[10] H.J. Oberle and K. Taubert, Existence and multiple solutions of the minimum-fuel orbit transfer problem. J. Optim. Theory Appl. 95 (1997) 243-262.

[11] L.T. Watson, A globally convergent algorithm for computing fixed points of $c^{2}$ maps. Appl. Math. Comput. 5 (1979) $297-311$.

[12] L.T. Watson, M. Sosonkina, R.C. Melville, A.P. Morgan and H.F. Walker. Algorithm 777: Hompack90: A suite of fortran 90 codes for globally convergent algorithms. ACM Trans. Math. Software 23 (1997) 514-549. 